\title{
Efectos de un Entrenamiento de Miembro Inferior con Res- tricción Parcial del Flujo Sanguíneo en la Fuerza Muscular y Biomarcadores Sistémicos de Daño Muscular e Inflamación
}

\author{
Effects of Lower Limb Training with Partial Restriction of Blood Flow on \\ Muscle Strength and Systemic Biomarkers of Muscle Damage and Inflammation
}

\author{
Carlos Bahamondes-Avila ${ }^{1,2}$; Jenny Lagos ${ }^{3,4}$; Luis Bustos M. ${ }^{3}$; José Alvarez-Castillo ${ }^{6}$;
} Francisco José Berral de la $\operatorname{Rosa}^{7}$ \& Luis A. Salazar ${ }^{3}$

BAHAMONDES-AVILA, C.; LAGOS, J.; BUSTOS, M. L.; ALVAREZ-CASTILLO, J.; BERRAL DE LA ROSA, F. J. \& SALAZAR, L. A. Efectos de un entrenamiento de miembro inferior con restricción parcial del flujo sanguíneo en la fuerza muscular y biomarcadores sistémicos de daño muscular e inflamación. Int. J. Morphol., 36(4):1210-1215, 2018.

\begin{abstract}
RESUMEN: El entrenamiento de fuerza, especialmente con alta intensidad de carga, permite aumentar la fuerza y trofismo muscular, pero también se asocia a daño muscular inducido por ejercicio (DMIE). Una nueva modalidad de entrenamiento, combina una baja intensidad de carga con la restricción parcial del flujo sanguíneo (RPFS) alrededor del músculo, siendo prometedor en cuanto el desarrollo de la fuerza y trofismo muscular. El objetivo del estudio fue comparar el rendimiento de fuerza máxima de los músculos cuádriceps e isquiotibiales (FM-Q y FM-I) y marcadores de daño muscular (CK) e inflamación sistémica (PCRus) entre un entrenamiento de baja intensidad de carga con RPFS, versus uno de alta y otro de baja intensidad de carga sin RPFS en jóvenes físicamente activos durante cuatro semanas de entrenamiento. Veintitrés participantes midieron la FM-Q y FM-I previo y al término de la intervención; además, antes del inicio de la primera sesión, y antes y después del término de la última sesión se midió la CK y PCRus. En los tres tipos de entrenamiento se produjeron aumentos equivalentes en la fuerza máxima, a excepción de la FM-Q del entrenamiento con baja intensidad sin RPFS. Solo en el entrenamiento con RPFS la CK y PCRus se modifican al finalizar la intervención, y aun cuando el estrés miocelular parece ser más alto que en los otros tipos de entrenamiento, no indicaría daño muscular.
\end{abstract}

PALABRAS CLAVE: Entrenamiento Kaatsu, Fuerza muscular, Daño muscular inducido por ejercicio.

\section{INTRODUCCIÓN}

El entrenamiento de fuerza $(\mathrm{EF})$ es una herramienta eficaz para estimular la hipertrofia y mejoras en la fuerza muscular (Kraemer et al., 2002), pero que también se asocia a un proceso de daño muscular inducido por ejercicio (DMIE); dentro de los biomarcadores séricos, la presencia de la enzima creatinquinasa (CK), en su isoforma CK-MM, propia del tejido muscular, indica DMIE (Brancaccio et al., 2008); asimismo, la proteina-C reactiva (PCR), producida en los hepatocitos en respuesta a la elevación de la interleucina 6, presenta una alta correlación con la inflamación sistémica (Michigan et al., 2011).

Una modalidad de EF, que combina una baja intensidad de carga (20-30 \% de una Repetición Máxima (RM)) con restricción parcial del flujo sanguíneo (RPFS) alrededor del músculo también ha evidenciado desarrollar la fuerza y el tamaño muscular, especialmente cuando se compara con el mismo ejercicio sin RPFS (Slysz et al., 2016), igualmente es efectivo en aumentar la masa muscular comparado con EF de alta intensidad (cargas superiores al $65 \%$ 1RM) (Lixandrão et al., 2018).

Dos factores principales que causan DMIE son el estrés mecánico y metabólico (Baird et al., 2012), siendo también los mismos mecanismos por los cuales el EF produce hipertrofia muscular (Schoenfeld, 2013). El estrés mecánico se genera con ejercicios de intensidad de carga sobre el $65 \%$ 1RM (EAI) y el estrés metabólico cuando la

\footnotetext{
${ }^{1}$ Escuela de Kinesiología, Universidad Mayor, Temuco, Chile.

${ }^{2}$ Doctorando en Ciencias, Universidad de Córdoba, Córdoba, España.

${ }^{3}$ Centro de Biología Molecular y Farmacogenética, Universidad de La Frontera, Temuco, Chile.

${ }^{4}$ Escuela de Tecnología Médica, Universidad Santo Tomás, Temuco, Chile.

${ }^{5}$ Departamento de Salud Pública, CIGES, Universidad de La Frontera, Temuco, Chile.

${ }^{6}$ Departamento de Educación, Universidad de Córdoba, Córdoba, España.

${ }^{7}$ Departamento de Deporte e Informática. Universidad Pablo de Olavide, Sevilla, España.
} 
BAHAMONDES-AVILA, C.; LAGOS, J.; BUSTOS, M. L.; ALVAREZ-CASTILLO, J.; BERRAL DE LA ROSA, F. J. \& SALAZAR, L. A. Efectos de un entrenamiento de miembro inferior con restricción parcial del flujo sanguíneo en la fuerza muscular y biomarcadores sistémicos de daño muscular e inflamación. Int. J. Morphol., 36(4):1210-1215, 2018.

carga es de baja intensidad (EBI) y realizados hasta el fallo muscular (Mitchell et al., 2012). En el caso del EF con RPFS existe un mayor estrés metabólico que mecánico, que cuya acción sinérgica mediaría otros mecanismos secundarios que favorecerían las adaptaciones en el desarrollo muscular y la fuerza (Pearson \& Hussain, 2014), no obstante, la evidencia sobre DMIE e inflamación es controversial, existiendo información dispar en cuanto a un mayor DMIE al compararse con sesiones aisladas o períodos de EAI o EBI (Wernbom et al., 2012).

El propósito del estudio fue comparar el rendimiento de fuerza máxima del músculo cuádriceps femoral (FMQ) y músculos isquiotibiales (FM-I), y el comportamiento de marcadores por DMIE e inflamación entre un EBI con RPFS, versus uno EAI y EBI sin RPFS en jóvenes físicamente activos durante cuatro semanas de entrenamiento.

\section{MATERIAL Y MÉTODO}

Participantes: Fueron reclutados varones de población universitaria, mediante una invitación abierta. Treinta y cinco voluntarios se ofrecieron a participar; cada uno de ellos firmó un consentimiento informado aprobado por el Comité de Ética de la Universidad Mayor, Chile. Para verificar los criterios de participación del estudio, se procedió a aplicar un cuestionario sobre el historial médico, la cuantificación del nivel de actividad física mediante el International Physical Activity Questionnaire (IPAQ), hábitos de entre- namiento y una evaluación en salud. Los criterios de inclusión consideraron ser deportista universitario recreacional y tener al menos 2 años de experiencia en EF; y los criterios de exclusión, ser deportista de rendimiento, poseer diagnóstico de enfermedades metabólicas o cardiorrespiratorias, hipertensión arterial, presencia de algún cuadro infeccioso o inflamatorio independiente de la causa, lesiones musculoesqueléticas, fumadores o estar ejecutando algún tipo de dieta. Finalmente, la muestra fue de 27 participantes. La Tabla I resume las características de los grupos de intervención.

Diseño de la investigación: Se formaron tres grupos de entrenamiento, mediante aleatorización simple: EBI con RPFS (RPFS), EAI y EBI, utilizando como criterio la FM-Q. Al inicio y término, se midió la FM-Q y la FM-I; además, antes del inicio de la primera sesión, y antes y después del término de la última sesión se midió la concentración sérica de CK) y de proteína C reactiva ultrasensible (PCR-us). Cada grupo tuvo 9 participantes, pero debido a inasistencias reiteradas, 4 sujetos se excluyeron, quedando 9, 6 y 8, respectivamente.

Intervención: Todos los participantes ejecutaron un entrenamiento de media sentadilla en prensa Smith y curl de femorales bilateral durante 12 sesiones en 4 semanas, distribuidos en 3 días alternos, en horario de 17:00 y 20:00 horas. El calentamiento consistió en 8 minutos de trote (8$11 \mathrm{~km} / \mathrm{h}$ ) y 3 minutos de elongación de tipo estática. Las características específicas de cada protocolo se describen a continuación y son resumidos en la Tabla II.

Tabla I. Característica de la muestra (media $\pm D S)$, según grupos de entrenamiento.

\begin{tabular}{lcccccc}
\hline & $\mathrm{N}$ & Edad (años) & IMC $\left(\mathrm{kg} / \mathrm{m}^{2}\right)$ & PS $(\mathrm{mm} \mathrm{Hg})$ & PD (mm Hg) & IPAQ (mets/min/sem) \\
\hline RPFS & 9 & $22,21 \pm 1,49$ & $25,77 \pm 2,78$ & $118,38 \pm 7,74$ & $75,88 \pm 8,46$ & $3625,56 \pm 1118,50$ \\
EAI & 6 & $22,02 \pm 2,46$ & $24,75 \pm 4,30$ & $120,33 \pm 11,27$ & $72,00 \pm 6,57$ & $3643,50 \pm 1948,37$ \\
EBI & 8 & $23,00 \pm 1,81$ & $25,42 \pm 3,14$ & $125,88 \pm 7,14$ & $79,25 \pm 13,13$ & $3585,31 \pm 838,36$ \\
& $p$ & 0,575 & 0,847 & 0,226 & 0,427 & 0,997 \\
\hline
\end{tabular}

Tabla II. Programas de entrenamiento para cada grupo.

\begin{tabular}{|c|c|c|c|}
\hline Grupo & RPFS & EAI & EBI \\
\hline$\%$ carga & $20 \% 1 \mathrm{RM}$ con RPFS & $70 \% 1 \mathrm{RM}$ & $20 \% 1 \mathrm{RM}$ \\
\hline S eries & 3 & 3 & 3 \\
\hline Repeticiones & $\begin{array}{l}\text { Al fallo muscular, como } \\
\text { máximo } 30\end{array}$ & $\begin{array}{l}12\left(1^{\circ}-6^{\circ} \text { sesión }\right) \\
15\left(7^{\circ}-12 \text { sesión }\right)\end{array}$ & Máximo 30 \\
\hline Micro Pausa* & 30 segundos & 60 segundos & 30 segundos \\
\hline Macro Pausa** & 60 segundos & 120 segundos & 60 segundos \\
\hline Presión de restricción $(\mathrm{mm} \mathrm{Hg})$ & $\begin{array}{c}160\left(1^{\circ}-6^{\circ} \text { sesión }\right) \\
180\left(7^{\circ}-12^{\circ} \text { sesión }\right)\end{array}$ & -- & ------ \\
\hline Ritmo de movimiento & $\begin{array}{l}2 " \text { concéntrico } \\
2 " \text { excéntrico }\end{array}$ & $\begin{array}{l}1 \text { " concéntrico } \\
1 \text { " excéntrico }\end{array}$ & $\begin{array}{l}\text { 2" concéntrico } \\
2 \text { " excéntrico }\end{array}$ \\
\hline
\end{tabular}

Abreviaturas: 1RM: resistencia máxima; RPFS: restricción parcial del flujo sanguíneo; EAI: entrenamiento de alta intensidad; EBI: entrenamiento de baja intensidad; * es el periodo de descanso que ocurre entre cada serie del mismo ejercicio, en este caso en la media sentadilla o en el curl de femorales. ** es el periodo de descanso que ocurre entre el cambio de ejercicio, en este caso entre la media sentadilla y el curl de femorales. 
BAHAMONDES-AVILA, C.; LAGOS, J.; BUSTOS, M. L.; ALVAREZ-CASTILLO, J.; BERRAL DE LA ROSA, F. J. \& SALAZAR, L. A. Efectos de un entrenamiento de miembro inferior con restricción parcial del flujo sanguíneo en la fuerza muscular y biomarcadores sistémicos de daño muscular e inflamación. Int. J. Morphol., 36(4):1210-1215, 2018.

Entrenamiento con Restricción Parcial del Flujo Sanguíneo (RPFS). Se desarrollaron ambos ejercicios con RPFS siguiendo recomendaciones técnicas previas (Slysz et al.); para aplicar la restricción se usó un manómetro de presión y dos manguitos neumáticos de $5,5 \mathrm{~cm}$ de ancho para cada miembro inferior, los cuales se instalaron en el muslo, $1 \mathrm{~cm}$ bajo el pliegue glúteo. La velocidad de movimiento fue guiada por un audio pregrabado. Luego del calentamiento, los manguitos se instalaron sin generar presión en los tejidos, fijándolos con velcro para mantenerlos en su lugar, inflándolos progresivamente a 160 ó $180 \mathrm{mmHg}$, respectivamente, manteniendo la presión constante durante toda la sesión, incluyendo los períodos de micro y macro pausa, y retirándose inmediatamente al término del entrenamiento. Durante todo el período de intervención no se modificó el \%1RM, solo la presión de restricción.

Entrenamiento de alta intensidad (EAI). Se utilizó un entrenamiento de sobrecarga siguiendo las recomendaciones para conseguir un aumento del trofismo y fuerza muscular (Kraemer et al.).

Entrenamiento baja carga (EBI). Este protocolo es similar al entrenamiento con RPFS, pero sin la aplicación de los manguitos neumáticos.

Variables: FM-Q y FM-I: Previo al inicio de las intervenciones, los participantes se ambientaron en las pruebas de media sentadilla en prensa Smith (FM-Q) y en curl de femorales en una camilla para músculos isquiotibiales (FMI); luego, en dos sesiones diferentes, se procedió a realizar las evaluaciones de FM-Q y FM-I, separadas entre sí por 72 hrs. Las pruebas fueron supervisadas por un solo miembro del equipo de investigadores, especializado en el EF. Previamente, el evaluador confirmó su error técnico de medición (ETM) en la prueba de media sentadilla, estableciendo un ETM absoluto $=2,7 \mathrm{~kg}$, ETM relativo $=3,4 \%$ y un ICC para ambas medidas de 0,94 . El test se aplicó con cargas progresivas submáximas durante un máximo de cinco intentos, en cada uno de ellos se buscaba realizar no más de cinco repeticiones, estas se consideraron válidas cuando se podía desplazar la carga en el rango definido, o sea, 0-90-0 ${ }^{\circ}$ de extensión, flexión y extensión de rodillas, respectivamente; con 5 minutos de pausa entre cada intento; de esta manera, se estimó la repetición máxima (1RM), de acuerdo a la siguiente ecuación:

$1 \mathrm{RM}=(100 \mathrm{x} \mathrm{kg}$ levantados en el último levantamiento válido) / (102,78 - 2,78 x Nº de repeticiones) (Brzycki, 1993).

Este procedimiento determinó las cargas individuales de EF por grupo muscular y grupos de intervención: RPFS: $20 \%$ 1RM, EAI: $70 \%$ 1RM y EBI: $20 \% 1$ RM.
CK y PCRus: Las muestras se obtuvieron por punción venosa en el antebrazo en tres momentos del plan de entrenamiento: $36 \mathrm{~h}$ antes del inicio (Pre S1), $36 \mathrm{~h}$ antes del término (Pre S12) y 60 h después del término (Post S12) de las intervenciones. A cada individuo se le extrajo $4 \mathrm{~mL}$ de sangre en un tubo sin anticoagulante. Los sueros fueron separados mediante centrifugación y almacenados a $-20^{\circ} \mathrm{C}$. Las cuantificaciones bioquímicas fueron realizadas en el analizador Humalyzer 3000 (Human, Alemania), utilizando control de calidad en dos niveles y calibradores. La actividad sérica de CK total se determinó mediante ensayo fotométrico cinético UV; y la concentración de PCRus se midió mediante ensayo inmunoturbidimétrico (Spinreact, S.A).

Análisis estadístico: el análisis descriptivo utilizó la media y desviación estándar; para verificar la distribución normal de la muestra se usó la prueba de Shapiro-Wilk; para las comparaciones en todas las variables, tanto al interior de los grupos, como entre ellos se realizó un análisis de modelo fijo de medidas repetidas de dos factores (grupo x tiempo), en dos momentos para FM-Q y FM-I y en tres para CK y PCRus, considerando de referencia la medición inicial de EAI. El nivel de significancia se estableció en $p<0,05$. El software utilizado fue Stata, versión 11.0.

\section{RESULTADOS}

Los valores (media \pm DS) de la FM-Q y FM-I se presentan en la Tabla III. Los principales resultados indican que en la RM-Q existieron diferencias significativas solo en la interacción del tiempo $(\mathrm{p}=0,01)$, presentándose cambios significativos en los grupos RPFS $(\mathrm{p}=0,01)$ y EAI $(\mathrm{p}=0,03)$. Para el RM-I, también los resultados muestran diferencias significativas en el tiempo $(p=0,00)$, en este caso en los tres grupos ( $\mathrm{p}=0,00$ para RPFS, 0,01 para HIT y 0,04 para LIT).

La CK se presenta en la Figura 1, los resultados de mayor relevancia indican que existieron diferencias significativas en la interacción grupo $\mathrm{x}$ tiempo $(\mathrm{p}=0,0175)$, ocurriendo en Post S12 entre RPFS e HIT $(p=0,002)$. Al analizar cada grupo, se observan estas diferencias solo en RPFS ( $\mathrm{p}=0,0003)$, ocurriendo entre Pre $\mathrm{S} 1$ y Pre $\mathrm{S} 12(\mathrm{p}=0,002)$ y entre Pre $S 1$ y Post $S 12(p<0,001)$. La PCRus se muestra en la Figura 2, existiendo sólo diferencias significativas en la interacción tiempo $(\mathrm{p}=0,0041)$. El análisis por grupo estas diferencias se presentan en RPFS ( $\mathrm{p}=0,0331)$ entre Pre $\mathrm{S} 1 \mathrm{y}$ Pre $\mathrm{S} 12(\mathrm{p}=0,014)$ y entre Pre $\mathrm{S} 12$ y Post $\mathrm{S} 12(\mathrm{p}=0,047)$. En las restantes interacciones no se observaron diferencias significativas. 
BAHAMONDES-AVILA, C.; LAGOS, J.; BUSTOS, M. L.; ALVAREZ-CASTILLO, J.; BERRAL DE LA ROSA, F. J. \& SALAZAR, L. A. Efectos de un entrenamiento de miembro inferior con restricción parcial del flujo sanguíneo en la fuerza muscular y biomarcadores sistémicos de daño muscular e inflamación. Int. J. Morphol., 36(4):1210-1215, 2018.

Tabla III. Valores (media \pm DS) de fuerza máxima de músculos cuádriceps (FM-Q) e isquiotibiales (FM-I) obtenidas al inicio y término de los protocolos de entrenamiento, incluyendo los valores $\mathrm{p}$.

\begin{tabular}{lccccc}
\hline Variable & $\begin{array}{c}\text { Tipo } \\
\text { entrenamiento }\end{array}$ & Inicio & Término & $\begin{array}{c}\text { Grupo - Tiempo - Grupo } \\
\text { x Tiempo }<0,05\end{array}$ & Tiempo x Grupo $<0,05$ \\
\hline \multirow{2}{*}{ FM-Q $(\mathrm{kg})$} & RPFS & $123,17 \pm 20,37$ & $132,25 \pm 17,40$ & & $0,01^{*}$ \\
& EAI & $109,05 \pm 7,43$ & $117,68 \pm 5,99$ & $0,09-0,01^{*}-0,07$ & $0,03^{*}$ \\
& EBI & $111,98 \pm 15,56$ & $111,38 \pm 13,25$ & & 0,75 \\
FM-I (kg) & RPFS & $94,41 \pm 26,58$ & $106,31 \pm 25,72$ & & $0,00^{*}$ \\
& EAI & $84,17 \pm 13,81$ & $101,76 \pm 24,62$ & $0,96-0,00^{*}-0,48$ & $0,00^{*}$ \\
& EBI & $84,44 \pm 20,13$ & $94,35 \pm 24,34$ & & $0,00^{*}$ \\
\hline
\end{tabular}

*: Cambios significativos.

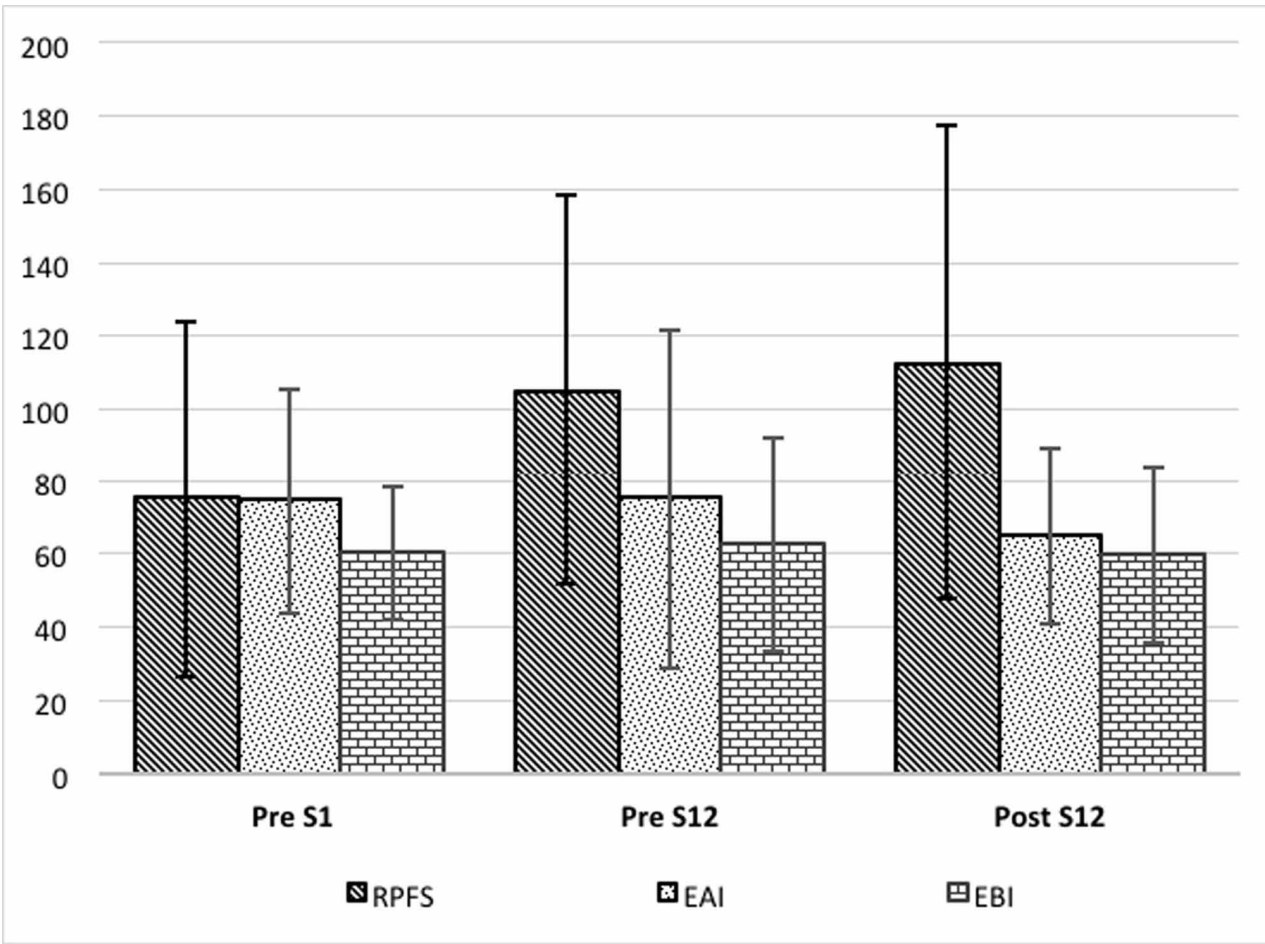

Fig. 1. Cambios en la actividad sérica de creatinquinasa $(\mathrm{CK})$ 36 horas previo al inicio de la intervención (Pre S1), 36 horas previo (Pre S12) a la última sesión y 60 horas post última sesión (Post S12). *: Cambios significativos intragrupo. **: Cambios significativos intergrupo.

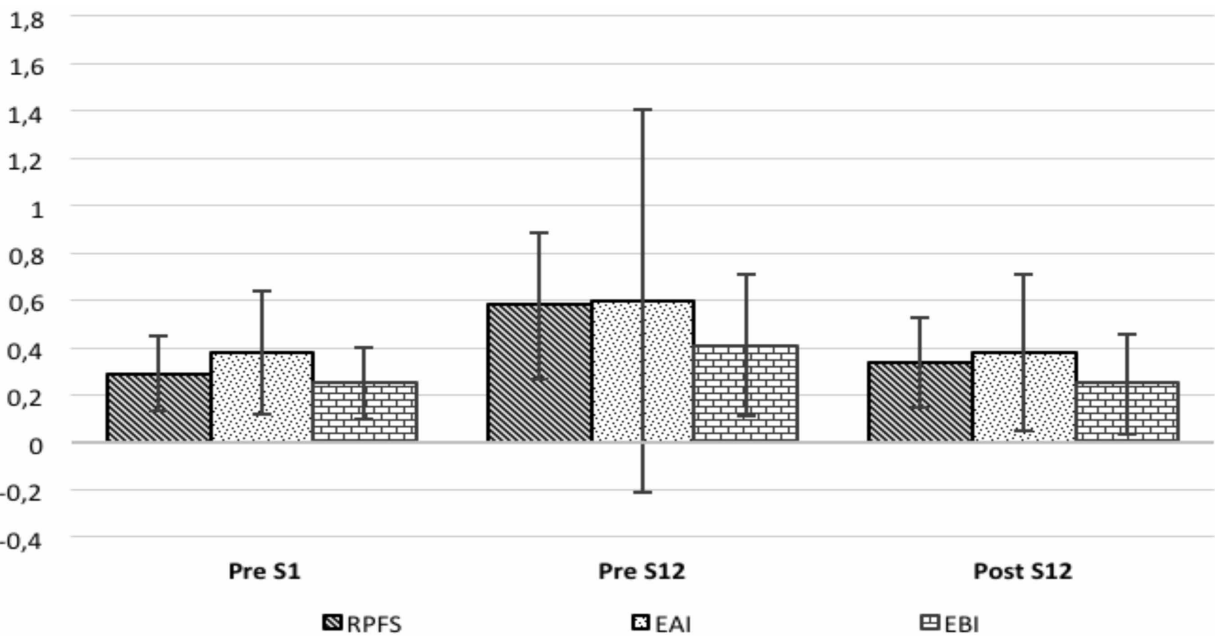

Fig. 2. Cambios en la concentración sérica de proteína $\mathrm{C}$ reactiva ultrasensible (PCRus) 36 horas previo al inicio de la intervención (Pre S1), 36 horas previo (Pre S12) a la última sesión y 60 horas post última sesión (Post S12). *: Cambios significativos intragrupo. 
BAHAMONDES-AVILA, C.; LAGOS, J.; BUSTOS, M. L.; ALVAREZ-CASTILLO, J.; BERRAL DE LA ROSA, F. J. \& SALAZAR, L. A. Efectos de un entrenamiento de miembro inferior con restricción parcial del flujo sanguíneo en la fuerza muscular y biomarcadores sistémicos de daño muscular e inflamación. Int. J. Morphol., 36(4):1210-1215, 2018.

\section{DISCUSIÓN}

El estudio demostró que en todos los entrenamientos aumentó la FM de ambos grupos musculares, excepto en la RM-Q de EBI, donde se mantuvo sin cambios, tampoco se presentaron diferencias al comparar los grupos con el EAI. La CK tiene una conducta distinta en cada grupo, en RPFS aumenta significativamente durante la intervención, en cambio, la estabilidad domina en EAI y EBI. Las diferencias entre grupos se producen entre RPFS y EAI al terminar la intervención. En la PCRus, el comportamiento de los grupos es el mismo, aumenta previo a terminar el entrenamiento, para luego disminuir, no obstante, solo se producen diferencias significativas al interior de RPFS.

En períodos de intervención menores a cuatro semanas, el resultado obtenido en el FM-Q de RPFS está en el rango de modificaciones observadas en deportistas (Cook et al., 2014). En cambio en la FM-I, el cambio fue menor al obtenido por varones no entrenados (Madarame et al., 2008). Estas ganancias de fuerza están en concordancia con observaciones previas durante este período de tiempo (Clark et al., 2011). Aun cuando se continúa discutiendo las implicancias fisiológicas, existe evidencia que indica una mayor señalización anabólica y síntesis proteica en solo una sesión de ejercicios con RPFS (Wernbom et al., 2013), lo cual podría justificar esta adaptación. Interesantemente, todos los grupos aumentaron su FM-I (11,74 a 20,9 \%), en magnitudes mayores a la FM-Q, probablemente se deba a que previo al inicio del EF los músculos isquiotibiales estaban relativamente débiles (Golik-Peric et al., 2011), este hallazgo es coherente con lo informado por los participantes durante la anamnesis, donde solo cuatro de ellos realizaban EF de esta musculatura más de una vez por semana. Aun cuando no se presentan diferencias significativas en la FM entre los grupos, los mayores cambios ocurren en el EAI (FM-Q + FM-I), donde interesantemente el volumen de entrenamiento (total de repeticiones $\mathrm{x}$ kg levantados) de este, es mayor en al menos $500 \mathrm{~kg}$ por sesión, lo cual contribuye a explicar este comportamiento. No obstante, es auspicioso obtener estos resultados con RPFS y EBI en solo cuatro semanas de entrenamiento, dado las menores intensidades relativas de carga y volumen aplicadas.

La mayoría de los estudios, indican que los ejercicios con RPFS no propician un mayor aumento en DMIE e inflamación que HIT o LIT, al compararse con LIT, no existen diferencias significativas entre los grupos, y se indica una tendencia a disminuir en la CK luego de finalizada una intervención con RPFS (Yasuda et al., 2014). Cuando el contraste es con HIT, la CK tampoco presenta diferencias significativas entre los grupos, aunque las mayores diferencias se dan en HIT (Clark et al.; Karabulut et al., 2013; Nielsen et al., 2017), contrariamente, la PCRus, a pesar de no producirse cambios significativos entre los EF, las mayores diferencias se dan en RPFS (Clark et al.). Estas diferencias con nuestros resultados podrían explicarse por la conjugación de las variables del EF con RPFS, pues se ha observado que estos indicadores parecen variar de acuerdo a la intensidad, duración o número de series (Sieljacks et al., 2016), tiempo de muestreo o modo de ejercicio (Karabulut et al.), ejecución de series al fallo vs series fijas (Thiebaud et al., 2013) o con compresión de los manguitos neumáticos continua vs intermitente (Neto et al., 2018); otro factor influyente podría estar en las características de la población de este estudio, que es joven y activa.

Incluir una medición mientras se desarrollaban los EF (Pre S12) tenía como propósito observar el efecto acumulativo de la carga de entrenamiento con recuperación parcial ( $<48$ h) sobre estos marcadores, observándose que solo el protocolo con RPFS produjo cambios significativos de la CK y PCRus, sugiriendo que el "efecto de combate repetido" (McHugh, 2003) permite una mayor tolerancia y adaptación en HIT y LIT; esta observación no la hemos visualizado en otros estudios, y aunque es difícil comprobar si estas diferencias en los biomarcadores se deban a condiciones especiales de este EF, como la compresión muscular inducida por el manguito de restricción, se hace necesario observar que los valores obtenidos de los biomarcadores se encuentran en un rango de normalidad (Pearson et al., 2003; Brancaccio et al.), aludiendo que el EF con RPFS genera un mayor estrés en la célula muscular, pero no propicia un mayor DMIE e inflamación en comparación con los otros protocolos usados. Parece relevante la presencia de DMIE e inflamación en las respuestas y adaptaciones musculares orientadas al desarrollo de la fuerza e hipertrofia; recientemente se ha indicado que en varones saludables, jóvenes y entrenados recreativamente, en un $\mathrm{EF}$ de tres semanas no parece haber daño celular general (daño muscular, inflamación o estrés oxidativo), pero si signos de inflamación tisular y estrés o reorganización focal de la membrana miocelular, que si estarían involucrados en la proliferación y aumento del contenido de células satélites miogénicas, favoreciendo una adaptación hipertrófica (Nielsen et al.).

En conclusión, los tres tipos de EF propiciaron aumentos equivalentes en la FM, a excepción de la FM-Q de EBI. Solo en el entrenamiento con RPFS, la CK y PCRus se modifican al finalizar la intervención, y aun cuando el estrés miocelular parece ser más alto que en los otros grupos, no indicaría DMIE. 
BAHAMONDES-AVILA, C.; LAGOS, J.; BUSTOS, M. L.; ALVAREZ-CASTILLO, J.; BERRAL DE LA ROSA, F. J. \& SALAZAR, L. A. Efectos de un entrenamiento de miembro inferior con restricción parcial del flujo sanguíneo en la fuerza muscular y biomarcadores sistémicos de daño muscular e inflamación. Int. J. Morphol., 36(4):1210-1215, 2018.

BAHAMONDES-AVILA, C.; LAGOS, J.; BUSTOS, M. L.; ALVAREZ-CASTILLO, J.; BERRAL DE LA ROSA, F. J. \& SALAZAR, L.A. Effects of lower limb training with partial restriction of blood flow on muscle strength and systemic biomarkers of muscle damage and inflammation. Int. J. Morphol., 36(4):1210-1215, 2017.

SUMMARY: Strength training, especially with high load intensity, allows increasing muscle strength and trophism, but it is also associated with exercise-induced muscle damage (EIMD). A new training modality, a combination of loading with the partial restriction blood flow (PRBF) around the muscle, being promising in the development of strength and muscular trophism. The aim of the study was to compare the maximum strength (MS) performance of quadriceps and hamstrings (MS-Q and MS-I) and muscle damage biomarkers (Creatine Kinase, $\mathrm{CK}$ ) and systemic inflammation (high sensitivity - CRP, hs-CRP) between a low intensity load training with PRBF, versus one high and another low load intensity without PRBF in physically active youngsters during four weeks of training. Twenty-three participants measured MSQ and MS-I and the intervention term. In addition, before the start of the first session, before and after the end of the last session, CK and hsCRP were measured. In the three types of training the equivalent benefits in MS are produced, an exception of the MS-Q of low intensity training without PRBF. Only in the training with PRBF, the CK and hs$\mathrm{CPR}$ are modified at the end of the intervention, and even though the myocellular stress seems to be higher than in the other types of training, it would not indicate muscle damage.

KEY WORDS: Kaatsu training; Muscle strength; Exercise induced muscle damage.

\section{REFERENCIAS BIBLIOGRÁFICAS}

Baird, M. F.; Graham, S. M.; Baker, J. S. \& Bickerstaff, G. F. Creatine-kinaseand exercise-related muscle damage implications for muscle performance and recovery. J. Nutr. Metab., 2012:960363, 2012.

Brancaccio, P.; Maffulli, N.; Buonauro, R. \& Limongelli, F. M. Serum enzyme monitoring in sports medicine. Clin. Sports Med., 27(1):1-18, 2008.

Brzycki, M. Strength testing-Predicting a one-rep max from reps-to-fatigue. $J$. Phys. Educ. Recreat. Dance, 64(1):88-90, 1993.

Clark, B. C.; Manini, T. M.; Hoffman, R. L.; Williams, P. S.; Guiler, M. K.; Knutson, M. J.; McGlynn, M. L. \& Kushnick, M. R. Relative safety of 4 weeks of blood flow-restricted resistance exercise in young, healthy adults. Scand. J. Med. Sci. Sports, 21(5):653-62, 2011.

Cook, C. J.; Kilduff, L. P. \& Beaven, C. M. Improving strength and power in trained athletes with 3 weeks of occlusion training. Int. J. Sports Physiol. Perform., 9(1):166-72, 2014.

Golik-Peric, D.; Drapsin, M.; Obradovic, B. \& Drid, P. Short-term isokinetic training versus isotonic training: effects on asymmetry in strength of thigh muscles. J. Hum. Kinet., 30:29-35, 2011.

Karabulut, M.; Sherk, V. D.; Bemben, D. A. \& Bemben, M. G. Inflammation marker, damage marker and anabolic hormone responses to resistance training with vascular restriction in older males. Clin. Physiol. Funct. Imaging, 33(5):393-9, 2013

Kraemer, W. J.; Adams, K.; Cafarelli, E.; Dudley, G. A.; Dooly, C.; Feigenbaum, M. S.; Fleck, S. J.; Franklin, B.; Fry, A. C.; Hoffman, J. R.; Newton, R. U.; Potteiger, J.; Stone, M. H.; Ratamess, N. A.; Triplett-McBride, T. \& American College of Sports Medicine. American College of Sports Medicine position stand. Progression models in resistance training for healthy adults. Med. Sci. Sports Exerc., 34(2):364-80, 2002.

Lixandrão, M. E.; Ugrinowitsch, C.; Berton, R.; Vechin, F. C.; Conceição, M. S.; Damas, F.; Libardi, C. A. \& Roschel, H. Magnitude of muscle strength and mass adaptations between high-load resistance training versus low-load resistance training associated with blood-flow restriction: a systematic review and meta-analysis. Sports Med., 48(2):361-78, 2018.

Madarame, H.; Neya, M.; Ochi, E.; Nakazato, K.; Sato, Y. \& Ishii, N. Crosstransfer effects of resistance training with blood flow restriction. Med. Sci. Sports Exerc., 40(2):258-63, 2008.

McHugh, M. P. Recent advances in the understanding of the repeated bout effect: the protective effect against muscle damage from a single bout of eccentric exercise. Scand. J. Med. Sci. Sports, 13(2):88-97, 2003.

Michigan, A.; Johnson, T. V. \& Master, V. A. Review of the relationship between C-reactive protein and exercise. Mol. Diagn. Ther, 15(5):265-75, 2011.

Mitchell, C. J.; Churchward-Venne, T. A.; West, D. W.; Burd, N. A.; Breen, L.; Baker, S. K. \& Phillips, S. M. Resistance exercise load does not determine training-mediated hypertrophic gains in young men. J. Appl. Physiol. (1985), 113(1):71-7, 2012.

Neto, G. R.; Novaes, J. S.; Salerno, V. P.; Gonçalves, M. M.; Batista, G. R. \& Cirilo-Sousa, M. S. Does a resistance exercise session with continuous or intermittent blood flow restriction promote muscle damage and increase oxidative stress? J. Sports Sci., 36(1):104-10, 2018.

Nielsen, J. L.; Aagaard, P.; Prokhorova, T. A.; Nygaard, T.; Bech, R. D.; Suetta, C. \& Frandsen, U. Blood flow restricted training leads to myocellular macrophage infiltration and upregulation of heat shock proteins, but no apparent muscle damage. J. Physiol., 595(14):4857-73, 2017.

Pearson, S. J. \& Hussain, S. R. A review on the mechanisms of blood-flow restriction resistance training-induced muscle hypertrophy. Sports Med., 45(2):187-200, 2014

Pearson, T. A.; Mensah, G. A.; Alexander, R. W.; Anderson, J. L.; Cannon, R. O. 3rd; Criqui, M.; Fadl, Y. Y.; Fortmann, S. P.; Hong, Y.; Myers, G. L.; Rifai, N.; Smith, S. C. Jr.; Taubert, K.; Tracy, R. P.; Vinicor, F.; Centers for Disease Control and Prevention \& American Heart Association. Markers of inflammation and cardiovascular disease: application to clinical and public health practice: A statement for healthcare professionals from the Centers for Disease Control and Prevention and the American Heart Association. Circulation, 107(3):499-511, 2003.

Schoenfeld, B. J. Potential mechanisms for a role of metabolic stress in hypertrophic adaptations to resistance training. Sports Med., 43(3):179-94, 2013.

Sieljacks, P.; Matzon, A.; Wernbom, M.; Ringgaard, S.; Vissing, K. \& Overgaard, $\mathrm{K}$. Muscle damage and repeated bout effect following blood flow restricted exercise. Eur. J. Appl. Physiol., 116(3):513-25, 2016.

Slysz, J.; Stultz, J. \& Burr, J. F. The efficacy of blood flow restricted exercise: A systematic review \& meta-analysis. J. Sci. Med. Sport, 19(8):669-75, 2016.

Thiebaud, R. S.; Yasuda, T.; Loenneke, J. P. \& Abe, T. Effects of low-intensity concentric and eccentric exercise combined with blood flow restriction on indices of exercise-induced muscle damage. Interv. Med. Appl. Sci., 5(2):539, 2013.

Wernbom, M.; Apro, W.; Paulsen, G.; Nilsen, T. S.; Blomstrand, E. \& Raastad, T. Acute low-load resistance exercise with and without blood flow restriction increased protein signalling and number of satellite cells in human skeletal muscle. Eur. J. Appl. Physiol., 113(12):2953-65, 2013.

Wernbom, M.; Paulsen, G.; Nilsen, T. S.; Hisdal, J. \& Raastad, T. Contractile function and sarcolemmal permeability after acute low-load resistance exercise with blood flow restriction. Eur. J. Appl. Physiol., 112(6):2051-63, 2012.

Yasuda, T.; Fukumura, K.; Fukuda, T.; Uchida, Y.; Iida, H.; Meguro, M.; Sato, Y.; Yamasoba, T. \& Nakajima, T. Muscle size and arterial stiffness after blood flow-restricted low-intensity resistance training in older adults. Scand. J. Med. Sci. Sports, 24(5):799-806, 2014

Dirección para correspondencia:

Prof. Dr. Luis Salazar Navarrete

Departamento de Ciencias Básicas

Facultad de Medicina

Universidad de La Frontera

Temuco - CHILE

Recibido : 15-06-2018

Aceptado: 07-08-2018

Email: luis.salazar@ufrontera.cl 